\title{
Katholische (Ex-)DDR-Medien in der Umstellung auf die Marktwirtschaft
}

\author{
von Renate Hackel-de Latour
}

In den Monaten seit der sanften Revolution haben sich die Ereignisse überschlagen, veränderte sich die politische Landschaft in atemberaubendem Tempo. Auch in der Medienlandschaft der DDR vollzog sich ein gewaltiger Umbruch. Über diese Medienlandschaft im früheren SED-Staat waren die westlichen Journalisten durchweg nur in groben Zügen informiert. Eine weitgehend unbekannte Größe war dabei die konfessionelle Presse, wie z. B. der katholische Medienmarkt. Es war ein kleiner Markt, der im „real existierenden Sozialismus" der DDR ein bescheidenes Dasein führte. Gleichwohl hatte die konfessionelle Publizistik eine Sonderstellung. Der Strudel der Veränderungen erfaßte auch die publizistische Arbeit der katholischen Kirche. Das enge Korsett, das der kirchlichen Medienarbeit in den vergangenen Jahrzehnten nur geringen Spielraum ließ, gibt es nicht mehr. Doch zuerst ein Blick zurück. Mit welchen Mitteln und unter welchen Bedingungen konnte die katholische Kirche in der Presse ihre Gläubigen informieren?

\section{Rückblick: Bemühungen um eine katholische Presse}

Die Kirchen in der DDR waren die „einzigen nichtsozialistischen und staatsfreien Organisationen" in der DDR. ${ }^{1}$ Damit waren auch die konfessionellen Presseorgane die einzigen Massenmedien in der DDR, die nicht von direkten inhaltlichen Vorgaben und personalpolitischen Einflußnahmen der SED betroffen waren. Die Kirchen wählten und bildeten ihre Redakteure in eigener Verantwortung aus.

Der Gründung des einzigen katholischen Verlages auf dem Gebiet der DDR, des 1951 in Leipzig gegründeten St. Benno-Verlages, waren jahrelange Verhandlungen vorausgegangen. Die weitestgehende Unterbindung des katholischen Medienschaffens war Ausdruck der prinzipiellen Religionsfeindlichkeit der marxistisch-leninistischen Weltanschauung.In den ersten Nachkriegsjahren war es den Katholiken in der DDR noch möglich, in bescheidenem Rahmen Kirchenzeitungen aus West-Berlin und den angrenzenden Diözesen Westdeutschlands zu beziehen. Zum 01.04.1949 wurde der Vertrieb verboten. Ein wenig gemildert wurde die Situation durch die beschränkte Möglichkeit, mehr oder weniger regelmäßig katholische Morgenfeiern über Radiosender zu verbreiten, sowie durch die Leistungen des eichsfeldischen Verlagshauses Cordier, dem in der Zeit des erfolglosen Bemühens um den St. Benno-Verlag die schwierige Aufgabe oblag, die Katholiken in der DDR mit dem notwendigsten religiösen Schriftum zu beliefern. Das wichtigste Kommunikationsmittel in diesen

Dr. Renate Hackel-de Latour ist Akademische Rätin am Diplom-Studiengang Journalistik der Katholischen Universität Eichstätt. 
Jahren war das gesprochene Wort - die Predigt und das Hirtenwort. Dieses publizistische Mittel gab der katholischen Kirche die Möglichkeit, zu drängenden Zeitfragen Stellung zu nehmen. Die Kanzel, also der kirchliche Verkündigungsort, übernahm in dieser bedrängten Zeit die „Funktion der Vermittlung aktueller ... universaler und sozialer Kommunikation“.2

\section{Kirchliche Verlags- und Medienarbeit in der DDR}

In den 50er Jahren gelang es der katholischen Kirche, für ihre damals in der DDR lebenden 2 Mio. Gläubigen (1990: 700.000 Katholiken) Lizenzen für Kirchenzeitungen, Amtsblätter und den St. Benno-Verlag zu erhalten. Staatliche Beschränkungen der katholischen Presse- und Verlagsarbeit gab es in mehrfacher Hinsicht: bei der Auflagenhöhe, der Papierzuteilung, beim Vertrieb und bei den Inhalten. Erschwert wurde die katholische journalistische Arbeit sicherlich nicht zuletzt aufgrund ihres Minderheitenstatus. Die Katholiken in der DDR waren eine „kleine Herde“, die gleichsam in einer doppelten Diaspora lebte: als Katholiken unter nichtkatholischen Christen und unter Nichtchristen.

So war auch der Grat der Möglichkeiten für einen katholischen Verlag, die Gläubigen mit religiösem Schrifttum zu versorgen, sehr schmal. Der St. Benno-Verlag war gemäß der Lizenzurkunde nicht nur für theologische Literatur und für religiöses Gemeindeschriftum zuständig, sondern auch für katholische Belletristik, für die Veröffentlichung alter und heutiger Werke der schönen Literatur und Kunst. ${ }^{3}$ In diesen Zuständigkeitsgrenzen beschränkte der St. BennoVerlag sein Themenspektrum auf das Gebiet der Verkündigung des Wortes Gottes, der Gottesdienstgestaltung, der Sakramentenspendung und des Gebetslebens, der Katechese und der religiösen Unterweisung, der theologischen Wissenschaft und Weiterbildung, des graphischen Schaffens, der Pflege der Kirchenmusik und der Dichtung aus christlicher Weltanschauung. ${ }^{4}$ Dies bedeutet, daß ein christlicher Verlag in der DDR auf rein christliche und kirchliche Themen beschränkt war. Die Behandlung von Fragen der Religionsphilosophie, Soziologie, Psychologie oder gar zeitgeschichtlicher Interpretationen war nicht erlaubt. ${ }^{5}$ Diese Themenbereiche waren den staatlichen Verlagen vorbehalten, denen es oblag, ,auf die Entwicklung einer vielseitigen, sozialistischen, schöngeistigen Literatur zu orientieren und insbesondere jene Werke zu fördern, die die Gegenwart im fortschrittlichen Geiste darstellen".6

Das Verlagsprogramm des St. Benno-Verlages enthielt $80-100$ Neuerscheinungen pro Jahr. Für jede Buchproduktion war eine Druckgenehmigung durch die "Hauptabteilung Verlage und Buchhandel“ nötig. Bei religiösen und liturgischen Schriften gab es die Genehmigung in der Regel ohne große Schwierigkeiten. Sobald es sich jedoch um Literatur handelte, die auch einen gewissen unterhaltenden Charakter aufwies, waren große Schwierigkeiten zu überwinden und zahlreiche Verzögerungen zu befürchten. Auch die Produktion christlicher Kinderbücher - mit Ausnahme von Büchern zur religiösen Unterweisung - war den kirchlichen Verlagen verwehrt, da volkseigene Kinderbuchverlage die jungen Leser mit Literatur versorgten. ${ }^{7}$ Ein Zugeständnis dieser Art wäre mit dem SED-Anspruch auf das staatliche Erziehungsmonopol nicht vereinbar gewesen. 
Im St. Benno-Verlag, einer $\mathrm{GmbH}$, in der alle katholischen Jurisdiktionsbezirke einen Gesellschafter stellten, erschienen auch die beiden katholischen Kirchenzeitungen, das Berliner 'St. Hedwigsblatt' (seit 1954) und der in Leipzig produzierte 'Tag des Herrn' (seit 1951). Ab Januar 1954 gab es für den Ostteil des Bistums Berlin das 'St. Hedwigsblatt', das wöchentlich in 8seitigem Umfang mit einer Auflage von 25.000 Exemplaren hergestellt wurde. Monatlich mußte der Leser dafür seit 195685 Pfennige bezahlen. Alle zwei Wochen kam der 'Tag des Herrn' in 100.000 Exemplaren bis Ende 1988 heraus. Er war für die sechs Jurisdiktionsbezirke außerhalb des Bistums Berlin bestimmt. Der Abonnementpreis für das Vierteljahr betrug 1,20 DM, der Einzelverkaufspreis20 Pfennige. Zum 01.01.1989 durfte die Kirchenzeitung bei gleichbleibender Druckauflage und unverändertem Umfang statt 14 tägig im wöchentlichen Rythmus erscheinen; eine Zielvorgabe, die bereits bei den Lizenzierungsverhandlungen Anfang der 50er Jahre an den Staat bestand.

Wodurch stellte sich nach Jahrzehnten bei der SED der Sinneswandel ein, den publizistischen Wünschen der katholischen Kirche nachzukommen? Die Ursache ist wohl in der vorsichtigen gesellschaftlichen Öffnung der katholischen Kirche zu sehen. Von Anfang an hielt sich die katholische Kirche in der DDR von gesellschaftlichen Aktivitäten fern und beschränkte sich auf Kultus und Caritas sowie Aufbau und Stärkung der Gemeinden. Schon in den frühen Jahren hatte sich die Berliner Bischofskonferenz für eine konsequente Verweigerung gegenüber den sozialistischen Medien entschieden, weil jede Aussage eines Kirchenvertreters zur Vortäuschung von Übereinstimmung, zur Vereinnahmung genutzt wurde. In den letzten Jahren gab es im Episkopat eine Wachablösung. Jüngere, in der DDR aufgewachsene Bischöfe haben darin inzwischen die Mehrheit. Für sie war das sozialistische System eine faktische Gegebenheit. Sie waren redefreudiger nach außen und offener im innerkirchlichen Dialog.

Für die Inhalte der Kirchenblätter hat sich dadurch nichts geändert. Sie durften nur über das Abonnement vertrieben werden, sie durften weder ausgetragen noch an den Kirchentüren oder an den Verkaufskiosken angeboten werden. Kein einziges Exemplar der Kirchenpresse konnte ohne Zustimmung des Presseamtes beim Vorsitzenden des Ministerrates in Umlauf gebracht werden. So war es möglich, die Auslieferung zu unterbinden bzw. die Redakteure zur Selbstzensur zu zwingen. Den Kirchenblättern drohten Maßregelungen, wie z. B. die Beschlagnahme der gesamten Ausgabe, wenn gesellschaftliche Themen zum Ausdruck kamen. Wenn kritische Anmerkungen abgedruckt wurden, so waren dies stets hochoffizielle kirchenamtliche Erklärungen und Weisungen. Von den Eingriffen der Zensurbehörde erfuhr der Abonnent einer Kirchenzeitung in der Regel nichts; es sei denn, das Kirchenblatt mußte Auflagenmeldungen des Presseamtes abdrucken. Nach der Veränderung der beanstandeten Stellen mußte die gesamte Auflage neu gedruckt werden. Bereits ab Ende der 50er Jahre mußten sich die Oberhirten, wenn sie zu gesellschaftspolitischen Fragen öffentlich Stellung nahmen, auf ihre ${ }_{n}$ klassischen" Kommunikationsmittel innerhalb der Kirche - Predigt und Hirtenwort - zurückziehen. Um den Lesern brisante Themen, die auch in der DDR diskutiert wurden, nahezubringen, fand die Kirchenpresse ein Schlupfloch. Nicht die eigenen Bischöfe kamen 
zu diesen Themen zu Wort, sondern vorsichtshalber der Vatikan, Bischöfe, Geistliche und Professoren aus anderen Ländern.

Ein pessimistisches Bild der Situation der katholischen Kirche in der DDR zeichnete der bereits verstorbene Theologieprofessor für Neues Testament in Erfurt, Wolfgang Trilling:

„Die katholische Kirche in der DDR lebt ohne kirchliche Öffentlichkeit. ... Unser Los und unsere Tugend ist das Schweigen. Schweigen ist eine Not, manchmal Schuld, aber es ist auch Wert. ... Die beiden Kirchenblätter bieten kein Forum für innerkirchliche Diskussionen. Von den Entwicklungen der Weltkirche sind wir fast abgeschlossen. Ihre Probleme, Nöte und Chancen berühren uns kaum, da wir nicht einbezogen werden. ${ }^{\text {" }}$

Neben diesen beiden offiziellen Informationsorganen erhielt der St. Benno-Verlag seit Februar 1952 die Lizenz für ein „Kirchliches Amtsblatt“, das in fünf unterschiedlichen Ausgaben 12mal jährlich in einer Auflage von 2.300 Exemplaren herauskam. Diese monatliche Veröffentlichung brachte vorwiegend Verlautbarungen des Heiligen Stuhls, Dekrete und Mitteilungen der jeweiligen Bischöfe sowie Beiträge von Theologen. Gemäß einer kirchlichen Bekanntmachung gestattete es der "vorgeschriebene Umfang des Blattes", dem Klerus darin wichtige Handreichungen für die Seelsorge zu bieten. ${ }^{9}$ Diese Handreichungen entwikkelten sich über die Jahre zu einer regelrechten Beilage namens „Pastorale Fragen" mit praxisbezogenen Beiträgen von Priestern aus der DDR oder auch aus anderen Ländern.

Von nur kurzer Lebensdauer war die Existenz der Zeitschrift "Christopherus. Monatszeitschrift der katholischen Jugend", für die der St. Benno-Verlag am 19.02.1952 die Lizenz erhielt. Nach 10 Heften mußte die Jugendzeitschrift auf Verfügung des „Amtes für Information" sein Erscheinen wieder einstellen; der amtlichen Begründung zufolge wegen Papiermangels. ${ }^{10}$ Die wahre Ursache dürfte jedoch im Alleinvertretungsanspruch der FDJ, die sich als einzig legale Vertreterin der Jugend in der DDR verstand, zu finden sein.

Noch vor der Lizenzierung des St. Benno-Verlages gab es für die katholische Presse ein wichtiges Datum. Im Herbst 1950 erteilte das „Amt für Information" der „Vereinigung katholischer sorbischer Geistlicher" die Lizenz für die Monatsschrift "Katolski Posol. Casopsis katolskich Serbow" (Katholischer Bote. Zeitschrift der katholischen Sorben). In der DDR lebten als einzige nationale Minderheit 50.000 Sorben, davon waren 15.000 Katholiken, denen anfangs monatlich später (ab 1955) 14tägig in knapp 3.000 Exemplaren der „Katholische Bote" zur Verfügung stand. Das Blatt beschäftigte sich ausschließlich mit konfessionellen Fragen; neben kommentierten Bibelstellen, Psalmen und Auslegungen wurden kirchliche Nachrichten, religiöse Gedichte $u$. a. abgedruckt. Das Blatt setzte keinen Bezug zur sorbischen Volkstumspolitik oder zum sonstigen aktuellen Geschehen. ${ }^{11}$ Bemühungen über das Presseamt beim Vorsitzenden des Ministerrates der DDR im Dezember 1976 um eine Erweiterung zum 8seitigen Wochenblatt sowie um die Anhebung der Auflage aufgrund wachsender Nachfrage wurden unter Verweis auf Papierknappheit kategorisch abgelehnt. 
Die Halbmonatsschrift erscheint ohne Mitwirkung kirchlicher Stellen. Die Zeitung hält sich selbständig und ohne hauptamtliche Mitarbeiter. Pfarrer Gerhard Werner aus Storcha ist bis heute (1991) im Nebenberuf Chefredakteur des „Katholischen Boten".

Außer jenen vom Staat lizenzierten Periodika hatte die katholische Kirche die Möglichkeit, für den „inneren Dienstgebrauch“, Rundschreiben etc. zu versenden oder zu verteilen. ${ }^{12}$ Mit dem Vermerk „Nur für innerkirchlichen Dienstgebrauch" konnten ohne staatliche Kontrolle hektographierte Texte, Materialien und Informationsdienste in Umlauf gebracht werden. Diese Schriften hatten erheblichen Einfluß auf den innerkirchlichen Informationsfluß.

Auf eine weitere Zeitschrift sei hier noch kurz hingewiesen: auf die katholisch firmierende „begegnung. Zeitschrift für Katholiken in Kirche und Gesellschaft". Das Konzept des Monatsblattes ging auf Walter Ulbrichts am 09.02.1961 eingeleiteten neuen religionspolitischen Kurs zurück. Ulbricht warb dabei um die kirchliche Mitarbeit beim Aufbau der sozialistischen Gesellschaft. Im Oktober 1961 kam die „begegnung" mit diesem Leitgedanken erstmals auf den DDR-Medienmarkt und bekundete ihr Ja zur sozialistischen Gesellschaft. Die katholischen Bischöfe in der DDR standen der „begegnung“ distanziert bis ablehnend gegenüber. Immer wieder forderte die Zeitschrift von den Bischöfen, sie sollten in der Öffentlichkeit ihre moralische Autorität in den Dienst der kommunistischen Friedenspolitik stellen. Die „begegnung“ setzte sich die selbstgestellte Aufgabe, „den Katholiken, die im realen Sozialismus der DDR leben und tätig sind, bei der allseitigen Praktizierung staatsbürgerlicher und christlicher Mitverantwortung für das gesellschaftliche Ganze eine konkrete Orientierungshilfe zu geben" ${ }^{13}$ Die Schrift erhielt finanzielle Unterstützung seitens des Staates. Ihre Auflage belief sich auf knapp 4.000 Exemplare. Im Gegensatz zu den Kirchenzeitungen konnte die „begegnung“ auch am Kiosk erworben werden. Neben der klaren einseitigen politischen Ausrichtung der "begegnung" und ihren vorwiegend parteitreuen Artikeln gab sie sich auch 'gut katholisch', und zwar sowohl im Bildteil als auch in den Beiträgen. Erbauliche Artikel über Prozessionen, Bistumstage, kirchliche Baudenkmäler, Bischofsjubiläen, die Weltkirche und den Vatikan hätten angesichts der geringen Auflage der kirchlichen Zeitungen 'St. Hedwigsblatt' und 'Tag des Herrn' durchaus eine Bereicherung sein können.

Eine Sonderstellung nahmen die Kirchen im Hörfunk ein. Keine der Blockparteien und keine der Massenorganisationen waren im Rundfunk mit Sendezeiten vertreten. Lediglich den Kirchen wurden für ihre Morgenfeiern wenngleich in bescheidenem Rahmen und zu nicht sehr günstigen Sendezeiten - ein Platz im Hörfunk eingeräumt. In einer Absprache mit der evangelischen Kirche einigte man sich auf ein Verhältnis von drei zu eins bei den Morgenfeiern. Bei hohen kirchlichen Feiertagen bildete sich ein alternierender Turnus heraus. Mithin konnte die katholische Kirche jährlich 18 Morgenfeiern, die sonntags in Radio DDR von 7.30 bis $8.20 \mathrm{Uhr}$ ausgestrahlt wurden, senden. 


\section{Veränderungen}

Nahezu zwei Jahrzehnte nach "Communio et Progressio" ist im Sommer 1988 eine Pressestelle beim Sekretariat der Berliner Bischofskonferenz errichtet worden. Ziel war die Verbesserung der innerkirchlichen Information, die Unterrichtung und Beratung der Bischöfe sowie die Unterrichtung kirchlicher und weltlicher Medien des In- und Auslandes über Vorgänge aus dem Leben der katholischen Kirche. Täglich gab die Pressestelle Meldungen und Berichte an die Medien weiter, die 14tägig in einem Pressedienst mit einer Auflage von 250 Exemplaren zusammengefaßt wurden. Ebenso sollten alle Jurisdiktionsbezirke regionale Pressestellen erhalten. Die Besetzung erwies sich als schwierig, da es an gut ausgebildeten Journalisten mit religiöser Überzeugung mangelt. Die Berliner Bischofskonferenz entschied erst in ihrer Vollversammlung am 04. und 05.12.1989 über die sechs Pressesprecher der Jurisdiktionsbezirke. Alle sind Geistliche.

Wie bereits erwähnt, erhielt der 'Tag des Herrn' in dieser Zeit die Bewilligung, wöchentlich zu erscheinen. Eine weitere Novität kam hinzu. Den sorbischen Christen wurde die Möglichkeit eröffnet, in ihrer Sprache religiöse Rundfunksendungen auszustrahlen. Seit November 1988 kamen im 2wöchigen Wechsel die katholische und die evangelische Kirche im Rahmen des sorbischsprachigen Programms des Senders Cottbus von Radio DDR II sonntags für 10 Minuten zu Wort.

Seit Oktober 1989 hat sich die katholische Medienarbeit geändert, neue Chancen boten sich, aber auch Schwierigkeiten bei der Umstellung auf die Marktwirtschaft stellten sich ein.

\section{Aufbruchstimmung}

Die katholische Kirche trat zunehmend aus ihrer selbstauferlegten medialen Verweigerung heraus und zeigte sich bei den sich abzeichnenden gesellschaftlichen Veränderungen engagiert. Nach der Wende wurden die Kirchen gebeten, bei der Demokratisierung der Gesellschaft mitzuwirken. Die noch ungewohnten veränderten Bedingungen dokumentierte Karl Heinz Ducke, Leiter der Studienstelle der Berliner Bischofskonferenz, in einer im Dezember 1989 unter dem Titel „Der Runde Tisch - Was will daran die katholische Kirche?" veröffentlichten Erklärung:

„Die katholische Kirche am Runden Tisch - das ist ein Risiko, wenn es nur Parteipolitik geben sollte; das ist eine Chance, wenn nicht nur Lösungen für aktuelle Probleme, sondern Fundamente einer erneuerten Gesellschaft gesucht werden; das ist schließlich auch eine Verpflichtung, die gesellschaftlichen Dimensionen des Christlichen zu verdeutlichen. Es geht uns um eine Gesellschaft, in der wir auch das wiederfinden, was unter Druck zur Gemeinschaft zusammengeführt hat, was uns kostbar ist. Wünschen wir uns eine Zukunft, an deren Gestaltung wir mitgearbeitet haben, in der wir unsere Hoffnungen wiedererkennen und sagen können: Das haben wir gewollt. ${ }^{14}$ 
Ebenso wirkte die katholische Kirche bei der Mediengesetzgebung und später im Medienkontrollrat mit. Im Dezember 1989 beschloß die Berliner Bischofskonferenz sieben Arbeitsgruppen zur Vorbereitung von Sachgesprächen mit staatlichen Gesprächspartnern zu bilden. Eine davon war die Arbeitsgruppe „Medienfragen" unter der Verantwortung von Bernhard Wiedemann, Redakteur der Pressestelle beim Sekretariat der Berliner Bischofskonferenz. Noch im 'selben Monat wurde eine Mediengesetzgebungskommission vom Ministerium der Justiz einberufen, zu der auch die Arbeitsgruppe "Medienfragen“ geladen war. Als Eckpunkte für einen Medienbeschluß forderte die katholische Vertretung:

- Meinungs- und Informationsfreiheit

- den Anspruch auf Gegendarstellung jedes von einer Veröffentlichung Betroffenen

- die Verankerung der Auskunftspflicht des Staates und der Behörden gegenüber den Medien

- die Pressetätigkeit von jeglicher Zulassung unabhängig zu machen

- die staatliche Zensur der Medien zu verbieten. ${ }^{15}$

In den Beschluß der Volkskammer der DDR über die Gewährleistung der Meinungs-, Informations- und Medienfreiheit vom 05.02.1990 waren diese Vorschläge eingegangen. Zudem wurde in dem 16-Punkte-Sofortmaßnahmenprogramm u. a. den Kirchen und Religionsgemeinschaften „das Recht auf angemessene Darstellung in den Medien zugesichert". ${ }^{16}$ Dies macht Schwierigkeiten, denn zum Erbe der jüngsten Vergangenheit in der DDR gehört, daß die staatlich ausgebildeten Journalisten über das Christentum und die Kirche völlig uninformiert sind und auf einen Personalbestand an katholischen Journalisten nicht zugegriffen werden kann.

In der Medienkontrollkommission, die die Einhaltung des Beschlusses überwachte, war die katholische Kirche durch Prälat Gerhard Lange vertreten. Über die endgültige Mediengesetzgebung sollte die aus freien und geheimen Wahlen hervorgegangene Volksvertretung entscheiden. Bei der Beratung wurde nach verschiedenen Sachbereichen - Printmedien, Rundfunk, Nachrichten- und Werbeagenturen - vorgegangen. Zu jedem dieser Sachgebiete wurde ein geeigneter Vertreter aus der Arbeitsgruppe "Medienfragen" benannt.

Nicht zufrieden war Berlins Bischof Sterzinsky mit dem Medien-Überleitungsgesetz. Er begrüßte zwar, daß den Kirchen darin angemessene Sendezeiten zugesichert wurden, der Entwurf lasse aber unklar, ob der öffentlich-rechtliche Rundfunk zur Einrichtung von Fachredaktionen für kirchliche Themen verpflichtet und ob den Kirchen die Verantwortung für Gottesdienstübertragungen und Verkündigungssendungen überlassen werde. Diese Punkte müssen nun in den Landesmediengesetzen geregelt werden.

\section{Neugründungen und Einstellungen}

Mit einer eigenen Wochenzeitung wollten katholische Laien ab Mitte Februar den Meinungs- und Willensbildungsprozeß in der DDR mitgestalten. Als 
Verleger fungierte die Paderborner Bonifatiusdruckerei mit ihrem Geschäftsführer Peter N. Kell. Ab Mai 1989 sollte das Blatt als Tageszeitung erscheinen. Nach sechs Ausgaben war die „Magdeburger - Orientierung zum Zeitgeschehen“ wieder verschwunden. Mit finanzieller Unterstützung des Erzbistums Paderborn brachte eine "katholische Koordinierungsgruppe" ein politisches Wochenblatt heraus. Mit einer Auflage von 100.000 Exemplaren und 8 Seiten Umfang war die Zeitung für 1 Ostmark zu haben. In der ersten Ausgabe hieß es zur Begründung für das gesellschaftliche Engagement, daß das Blatt Information und Orientierung für die Katholiken sein wolle, aber auch für andere Christen und alle Suchenden in der Umgestaltung „unserer Verhältnisse zu einer menschenwürdigen Demokratie“. Als Ursache für das schnelle Ende nannte die Redaktion, daß sich die grundlegende Zielrichtung der Publikation, eine „Orientierung zum Zeitgeschehen" im Vorfeld der Volkskammerwahlen zu geben, mit dem vorverlegten Wahltermin erledigt habe. Ferner wurden für die Einstellung „unübersehbare Probleme" angeführt. Die Konkurrenz westlicher Presseorgane, der schwierige Vertrieb über die Pfarrämter und die Überforderung der nebenberuflichen Redakteure hätten eine weitere Produktion unmöglich gemacht. Dennoch sei der Versuch, „mit unterschiedlichen Fähigkeiten und Ansichten eine gemeinsame Idee zu verwirklichen", geglückt. In einem Schreiben an die Abonnenten der Zeitung wünschen die Herausgeber "für den Weg in die Demokratie" allen „viel Phantasie und Engagement, Toleranz und aktives Mittun“. ${ }^{17}$

Die gesellschaftspolitische Veränderung in der DDR brachte auf der anderen Seite das 'Aus' für einige Publikationen. Darunter war auch die Zeitschrift „begegnung“, die die kirchenpolitische Linie der DDR-CDU - und damit der SED - gegenüber der christlichen Bevölkerung vertrat. Die Monatsschrift konnte wie andere Medien und Medieninstitutionen - nicht kostendeckend wirtschaften. Über den mittlerweile aufgelösten Nationalrat der Nationalen Front - den Zusammenschluß der ehemaligen Blockparteien und „Massenorganisationen“ erhielt die „begegnung" einen jährlichen Zuschuß von 90.000 DDR-Mark zu den Gehaltskosten. Das Ausbleiben des Geldes brachte für die Zeitschrift das Ende. In der letzten Ausgabe, dem Dezemberheft 1990, hatte das Blatt noch versucht, sich an die "Wende" anzuhängen. Wie sich zeigte, erfolglos. In der kurzen Meldung heißt es: „Das Kollegium erörterte auch die weitere Grundlinie der Zeitschrift und ermutigte dazu, die demokratische Neuentwicklung in unserem Staat mitzutragen". ${ }^{18}$ Eine Kehrtwendung machte die "begegnung" auch in ihrem Verhältnis zur katholischen Amtskirche. Während sie über Jahrzehnte die Kirche schulmeisterlich belehrte, sie solle sich dem 'friedenspolitischen Engagement der SED' anschließen, spricht sie nun Dank aus "für die ermutigende Haltung der Kirchen, die mit ihren nachdrücklichen Forderungen z. B. nach Abbau von Feindbildern, nach Respektierung des Ersterziehungsrechts der Eltern und nach gleichen Berufs- und Bildungschancen für alle Bürger dringende Fragen unserer Zeit im Bewußtsein der Öffentlichkeit wachgehalten haben“. ${ }^{19}$ In der "begegnung“ wurden diese Probleme nie thematisiert. Noch im Abschiedsbrief an die Leser verdreht und beschönt „begegnung“-Chefredakteur Horst Walligora die Berichterstattung der Zeitschrift in den 28 Jahren ihres Erscheinens. Er behauptet in dem Schreiben, die „begegnung“ sei ein „unverwechselbarer Teil unserer Medienlandschaft" gewesen, "weil sie Zeichen katholischer Präsenz in unserem Lande setzte und Zeugnis von der Pluralität katholischen Lebens ablegte." Er 
schließt mit den Worten: „Wir würden uns freuen, wenn das Anliegen der 'begegnung', kritisch-ausgewogen zur Entwicklung in Kirche und Gesellschaft Stellung zu beziehen, durch Sie weitergetragen und lebendig gehalten würde". ${ }^{20}$

Neben den zahlreichen Institutionen, die noch zu DDR-Zeiten verschwanden, gehörte das Amt für Kirchenfragen des Ministerrates der DDR zum 30.09.1990. Nach sowjetischem Vorbild wurde 1957 in der DDR die Funktion eines Staatssekretärs für Kirchenfragen geschaffen. Die ihm beigegebene Behörde war das Amt für Kirchenfragen. Über dieses Amt wurden alle Kontakte zwischen der DDR-Regierung und den Kirchen abgewickelt. Während es sich bei den Staatssekretären stets um hochrangige SED-Mitglieder handelte, kamen die Stellvertreter aus den Reihen der Blockpartei CDU. Das Amt war dem Vorsitzenden des Ministerrates unterstellt, es war aber nicht das Organ zur Formulierung der Kirchenpolitik, sondern das Instrument zu ihrer Durchsetzung. Grundsätzliche Fragen des Verhältnisses zwischen Staat und Kirche wurden von der Arbeitsgruppe „Kirchenfragen" beim Zentralkomitee der SED oder, wenn nötig, vom Generalsekretär der SED selbst entschieden. Für die katholische Kirche in der DDR war das Amt für Kirchenfragen die Verbindungsstelle zur Staats- und Parteiführung, mit der sie in der Regel jeden direkten Kontakt mied.

Vorbei war es mit der Nummer 15/1990 vom 26.11.1990 auch mit den „Informationen - Berichten - Materialien aus dem Bereich der Berliner Bischofskonferenz". Dies ist eine Folge des Zusammenschlusses der Berliner und der Deutschen Bischofskonferenz. Das bisherige Sekretariat der Berliner Bischofskonferenz wird als Außenstelle des Sekretariats der Deutschen Bischofskonferenz weitergeführt. Mit der Beendigung der Sekretariatsarbeit endete auch die Arbeit der Pressestelle. Um weiter aus dem Leben der katholischen Kirche aus dem Bereich der ehemaligen Berliner Bischofskonferenz journalistisch berichten zu können, wurde beschlossen, bei der Außenstelle Berlin des Sekretariats der Deutschen Bischofskonferenz ein Referat für Presse- und Öffentlichkeitsarbeit zu errichten.

\section{Kooperationen}

Der Wind des freien Wettbewerbs traf auch die konfessionelle Publizistik. Den Bistumsblättern mangelt es vor allem an Erfahrungen in den Bereichen der Vertriebswerbung sowie des Anzeigenverkaufs. Bis zur Wende hatte der SEDStaat ihnen weitgehend die wirtschaftliche Verantwortung abgenommen, indem er die Herstellungskosten der Publikationen bis zu drei Fünfteln subventionierte und eine feste Auflage garantierte, ob sie verkauft wurde oder nicht. Ein weiteres Problem kommt hinzu: Der 'Tag des Herrn' kennt seine Abonnenten nicht. $\mathrm{Zu}$ den Kontrollmechanismen in der DDR gehörte es auch, daß die Deutsche Post den Verlagen die Auflage komplett abnahm und auch die Abonnentenadressen verwaltete. Wahrscheinlich wird die Deutsche Post dieses Jahr die Adressen an die Verlage verkaufen.

Ablehnend reagierte der Chefredakteur des 'Tag des Herrn', Gottfried Swoboda, in der Nummer 13/1990 vom 01.04.1990 in einem Beitrag unter der 
Überschrift "Ohne Rücksicht auf Verluste“ auf den Versuch westlicher Bistumszeitungen und Missionszeitschriften, in der DDR zu werben:

„Unter dem Motto 'Glauben über Grenzen hinweg' wirbt seit geraumer Zeit die Paderborner Kirchenzeitung 'Der Dom' in halbseitiger Aufmachung um Geschenk-Abonnements für Katholiken in der DDR. ... Unter dem Deckmantel der christlichen Nächstenliebe versucht man so, seine Marktinteressen zu verstecken. Die DDR mag zwar wirtschaftlich am Boden sein, aber kirchlicherseits erwies sich die hiesige Pastoral trotz der kurzen Personaldecke als durchaus eigenständig und lebendig“. ${ }^{21}$

Auch inhaltlich sind die Kirchenzeitungen vor neue Aufgaben gestellt worden. So müssen sie sich jetzt mit Themen befassen, die früher aufgrund der Pressezensur nicht behandelt werden durften oder aber erst mit der Öffnung der Grenzen aktuell geworden sind, wie z. B. die in die DDR drängenden Jugendsekten und New-Age-Gruppen. Als besonderes Anliegen seiner Redaktion nannte Swoboda die Unterstützung der Katholiken in ihrem Einsatz für die Gesellschaft und die Begleitung der neugegründeten katholischen Verbände. Außerdem will sich das Blatt bemühen, ein Stück DDR-Geschichte aufzuarbeiten.

Obwohl es sich Chefredakteur Swoboda persönlich sicherlich anders gewünscht hätte, mußte auch der 'Tag des Herrn' mit westdeutschen Bistumsblättern kooperieren. Seit der Weihnachtsausgabe arbeitet das Blatt in der sog. Nordschiene, den Bistumszeitschriften von Berlin/West (Petrusblatt, Morus-Verlag), Hildesheim (Kirchenzeitung Hildesheim, Bernward-Verlag) und Osnabrück (Verlag des Kirchenboten Osnabrück) mit. Die Einbindung des 'Tag des Herrn' in die Nordschiene, die damit ihre Auflage auf 180.000 erhöht, zieht eine Fülle von Konsequenzen nach sich: Der Umfang des Blattes erweitert sich von 8 auf 24 Seiten, das Format wird vergrößert und dem der drei Partnerzeitungen angeglichen. Für die 72.000 Abonnenten des 'Tag des Herrn' erhöht sich der Preis von 20 auf 90 Pfennig pro Nummer. Mit diesem Preis kann sich die Zeitung selber tragen - sofern die Auflage nicht weiter sinkt. Herausgeber der Wochenzeitung sind - entgegen den ursprünglichen Absichten - nicht alle Jurisdiktionsbezirke. Bischof Sterzinsky beteiligte sich mit seiner Diözese nicht an der Herausgeberschaft. Für die zukünftige Ost-West-Zusammenarbeit mit der Nordschiene gab Swoboda im 'Tag des Herrn' zu bedenken:

„Die Leser der Kooperation können voneinander lernen, dabei verbindet die gemeinsame Diasporasituation. Der 'Tag des Herrn' wäre dann eine Art Brücke beim Zusammenwachsen der durch unterschiedliche Systeme geprägten Menschen". ${ }^{22}$

Der 'Tag des Herrn' erscheint als überregionale Kirchenzeitung in ihrem bisherigen Verbreitungsgebiet mit diözesanen Wechselseiten. Swoboda setzt auf die Zusammenarbeit mit den Pressereferenten in den Jurisdiktionsbezirken, die wöchentlich Stoff für die 8 Regionalseiten des Blattes liefern sollen. Mit der neuen Struktur könne der 'Tag des Herrn' die seelsorgliche Arbeit und das Engagement vieler Christen in Gemeinde, Politik und Gesellschaft weitaus in- 
tensiver begleiten und in erheblich größerem Umfang Glaubenshilfe für Familie, Jugend und Kinder bieten. 12 der 24 Seiten werden in gemeinsamer Verantwortung und mit gleichem Inhalt von den vier gleichwertigen Kirchenzeitungspartnern produziert und haben $u$. a. folgende Schwerpunkte:

- Vorbereitung auf den Sonntag und Impulse aus dem Glauben;

- Berichte und Informationen aus der Weltkirche;

- Reportagen über Aktivitäten der Christen in Deutschland und aus anderen Ländern;

- je eine Seite für die Familie und die Kinder;

- auch die Unterhaltung soll nicht zu kurz kommen. ${ }^{23}$

Gedruckt wird in Osnabrück. Der 'Tag des Herrn' erscheint im ebenfalls neukonzipierten St. Benno-Verlag.

Synchron zur Neugestaltung des 'Tag des Herrn' erfolgte die Vereinigung der beiden Berliner Kirchenzeitungen. Das 'Petrusblatt' und das 'St. Hedwigsblatt' gaben als Weihnachtsausgabe eine Doppelnummer heraus, erstmals als gemeinsame 'Katholische Kirchenzeitung für das Bistum Berlin'. Für die Bezieher im Gebiet der früheren DDR wird sie 90 statt der bisherigen 85 Pfennige kosten. Im Untertitel werden die beiden früheren Namen weitergeführt. Zum geistlichen Beirat der Zeitung ernannte der Bischof von Berlin, Georg Sterzinsky, den Chefredakteur des 'St. Hedwigsblattes' und Berliner Ordinariatsrat, Prälat Gerhard Lange. Die neue Kirchenzeitung beteiligt sich an der Nordwestkooperation mit Hildesheim und Osnabrück.

Bald nach der Öffnung der Grenzen im November 1989 stellten sich die Gesellschafter des St. Benno-Verlages die Frage nach dem Fortbestand des Verlages unter den neuen Bedingungen. Die bald darauf einsetzende Konkurrenz durch westliche Verlage verschärfte die Lage unter den Aspekten der freien Marktwirtschaft. Auf ihrer Vollversammlung am 11./12.06.1990 in Berlin beschlossen die Bischöfe der Berliner Bischofskonferenz, daß der St. Benno-Verlag weiterhin als regionaler Verlag für den Bereich der DDR katholische Schriften verlegen soll, vorbehaltlich einer 75-\%igen Beteiligung der westdeutschen Verlage. Umgehend sollte der Verlag Gespräche zur Kooperation und Kapitalbeteiligung unter marktwirtschaftlichen Gesichtspunkten mit dem BernwardVerlag (Hildesheim), dem Morus-Verlag (Berlin/West) und dem Weltbild-Verlag (Augsburg) aufnehmen.

Am 03.09.1990 entschieden sich die Bischöfe der Berliner Bischofskonferenz für die Neugründung eines Buch- und Zeitschriftenverlages, der einerseits den veränderten Verhältnissen im vereinten Deutschland entspricht, andererseits aber der Tradition des St. Benno-Verlages verpflichtet bleibt.

Mit mehrheitlich westlichem Kapital wurde am 06.12.1990 in München die 'St. Benno Buch- und Zeitschriften-Verlagsgesellschaft mbH' gegründet. Neue Mehrheitsgesellschafter sind der Hildesheimer Bernward-Verlag, der Berliner Morus-Verlag und der Verlag des Kirchenboten Osnabrück. Die drei Verlage bringen jeweils $100.000 \mathrm{DM}$ in das Stammkapital von $400.000 \mathrm{DM}$ für die 
neue St. Benno-Verlagsgesellschaft ein. Den Rest teilen sich zu gleichen Teilen die Diözesen Dresden-Meißen, die Apostolische Administratur Görlitz sowie die Bischöflichen Ämter Erfurt-Meiningen, Magdeburg und Schwerin. Klaus Behnke, Diplom-Kaufmann, 33, wurde zum neuen Geschäftsführer ernannt. Er war zuvor in leitender Tätigkeit beim Kevelaerer Verlagshaus Butzon \& Bercker für den Buchvertrieb tätig. Zum 01.01.1991 nahm die neue Gesellschaft in Leipzig ihrè Geschäfte auf. Wie auch in anderen Medienbereichen der DDR gehen Strukturveränderungen mit Entlassungen einher. Von den 70 Mitarbeitern können maximal 20 weiterbeschäftigt werden. Für die ausscheidenden Mitarbeiter übernehmen die Bischöfe der 'Region Ost' den Sozialplan. Die große Zahl der Verlagsangestellten hing außer mit der in der DDR üblichen Arbeitsproduktivität auch damit zusammen, daß die Kirche in der sozialistischen Gesellschaft für nicht wenige Christen Arbeitsmöglichkeiten schaffen wollte.

Der neue St. Benno-Verlag gibt weiterhin den 'Tag des Herrn' heraus. Im Buchvertrieb will der Verlag an dem guten Ruf des Vorgängers für religiöse Literatur anknüpfen. Verstärkt hinzukommen sollen Kinderbücher, Sachbücher und Regionalia.

\section{Engagement im Rundfunk}

Bereits mehrfach erwähnt wurde die dünne Personaldecke der katholischen Kirche der DDR im Medienbereich. Die wenigen im katholischen Printbereich tätigen Redakteure hatten ihr Handwerk nach dem Prinzip 'learning by doing' erlernen müssen; vom Rundfunk ganz zu schweigen. Der Deutsche Fernsehfunk erhielt zum Ostersonntag 1990 eine Kirchenredaktion. Ein Teil der Sendezeit stand der katholischen Kirche zu. Da keine ausgebildeten Journalisten zur Verfügung standen, um kirchliche Nachrichtensendungen auszustrahlen, wurden Gottesdienste übertragen. Zusätzlich erhielten die katholische und evangelische Kirche im Wechsel bei den Regionalsendern, z. B. bei Radio Mecklenburg-Vorpommern die Möglichkeit zur Ausstrahlung von Morgenandachten. Ab September wurde jeden Mittwoch von 18.50 bis 19.00 Uhr eine kirchliche Nachrichtensendung, Veranstaltungshinweise, Servicemeldungen und Nachrichten über Aktivitäten im katholischen Bereich gebracht. Verantwortlich für den Inhalt der Sendungen ist der Rundfunkbeauftragte des Bischöflichen Amtes Schwerin, Matthias Baerens.

In einem gemeinsamen Brief wandten sich die Berliner Bischofskonferenz, der Bund der Evangelischen Kirchen und die Arbeitsgemeinschaft Christlicher Kirchen am 24.09.1990 an die Direktoren der Landessender und Intendanten des DDR-Hörfunks und Fernsehens. In diesem Schreiben meldeten die Kirchen und Religionsgemeinschaften ihren Bedarf an Sendungen mit spezifisch kirchlicher bzw. religiöser Thematik an. Ebenso wird die Weiterführung von Gottesdiensten, Morgenfeiern und ähnlichen Sendungen gewünscht. Die Kirchenvertreter äußerten ihre Sorge, daß mit der Aufsplitterung der vorhandenen zentralen Frequenzketten besonders diese Sendungen in Frage gestellt würden. Im Rahmen des 'Demokratisierungsprozesses' des Rundfunks sei es gelungen, Kirchenredaktionen einzurichten und kirchliche Sendungen zu installieren. Der 
Brief schließt mit der Bitte, bei allen Vereinbarungen zwischen den Landesrundfunkanstalten die Anliegen der Kirchen und Religionsgemeinschaften ausreichend zu berücksichtigen. ${ }^{24}$

Bis zum Sommer sollen die gesetzlichen Rahmenbedingungen für den Rundfunk in den neuen Bundesländern feststehen. Nach dem derzeitigen Diskussionstand haben die Kirchen gute Ausgangsbedingungen, als Mitglieder in die Rundfunkräte der öffentlich-rechtlichen Anstalten sowie in die Aufsichtsbehörden des kommerziellen Rundfunks zu kommen. Sicherlich werden ihnen auch angemessene Sendezeiten eingeräumt. Wie es mit der Einrichtung von Kirchenredaktionen bestellt sein wird, bleibt abzuwarten.

\section{Aus- und Weiterbildung von Journalisten}

Mit Schnell- und Kurzkursen zum Erwerb journalistischer Grundkenntnisse bemüht sich die katholische Kirche in der DDR, die Teilnehmer dazu zu befähigen, qualifizierte Beiträge über das kirchliche Leben zu schreiben oder für den Hörfunk zu produzieren. Hilfe kommt auch von der 'Arbeitsgemeinschaft Katholische Presse' (AKP), die eine gemeinsame Aus- und Weiterbildung von Redaktions- und Verlagsmitarbeitern aus West und Ost vorsieht. Zudem haben die Redakteure aus der Ex-DDR Nachholbedarf. Sie können nicht davon ausgehen, daß die Leser mit der traditionellen Gestaltungsweise der Kirchenzeitung auch künftig zufrieden sind. Die Erstellung zeitgerechter Layouts sowie der Einsatz moderner Technik ist gewiß mitentscheidend für den künftigen Erfolg der konfessionellen Presse - ein Austausch von Redakteuren nach hüben und drüben beschleunigt diesen Lemprozeß.

\section{Verein für kirchliche Medienarbeit in Osteuropa}

Um die "dauerhafte Einrichtung europäischer Medien-Zusammenarbeit aus christlichem Geist" aufzubauen, gründeten katholische Verleger und Medienfachleute aus neun Staaten im Juni 1990 den 'Verein zur Förderung religiöser Medienarbeit in Mittel- und Osteuropa'. ${ }^{25}$ Die finanziellen Mittel des Vereins sollen ausschließlich zur Förderung des katholischen Medienwesens in den ostmitteleuropäischen Ländern dienen. Sitz des internationalen Vereins ist Wien. Initiator war der Generaldirektor des Grazer Styria-Verlages, Hanns Sassmann.

Die konkrete Arbeit des internationalen Vereins soll im Sinn einer überregionalen 'Medien-Dienstleistungsgesellschaft' in Fachkommissionen geleitet werden, die international zusammengesetzt sind: Polen, Ungarn, CSFR (Böhmen/Mähren und Slowakei), Jugoslawien (Slowenien und Kroatien), Rumänien. Katholische Verlage und Institutionen in Österreich, Deutschland und der Schweiz wollen mit ihren Mitarbeitern für die katholischen Kollegen aus Ostund Mitteleuropa "Hilfe zur Selbsthilfe leisten" ${ }^{26}$ Im einzelnen sind folgende Fachkommissionen vorgesehen: Zeitungen und Zeitschriften, Buch, technische Entwicklung, elektronische Medien, Nachrichtenagenturen, Aus- und Weiterbildung. Der Medien-Förderverein bereitet einen von der österreichischen Bun- 
desregierung finanzierten Lehrgang für osteuropäische Nachwuchsjournalisten an der Katholischen Universität Lublin in Polen vor. Ein drängendes Anliegen, angesichts der Zahl der Neugründungen katholischer Periodika in diesen Staaten. Langfristig soll es dabei nicht um "einseitige und einmalige Aktionen zwischen großzügigen Gönnern und bescheidenen Beschenkten gehen", so Frantisek Sykora, der Vorsitzende des Vereins. Es gehe darum, die „positiven und kreativen Kräfte aus Ost und West zu vereinen“, um die europäischen Veränderungsprozesse in der Besinnung auf die gemeinsamen christlichen Wurzeln zu beeinflussen. ${ }^{27}$

\section{Anmerkungen:}

1 DDR-Handbuch, Hg. vom Bundesministerium für innerdeutsche Beziehungen, Köln $1979,588$.

2 Christoph Klessmann/Frank Pingel (Hg.), Gegner des Nationalsozialismus. Wissenschaftler und Widerstandskämpfer auf der Suche nach der historischen Wirklichkeit, Frankfurt/M.-New York 1980, 133

3 St. Benno-Verlag (Hg.), Gesamtverzeichnis 1951 - 1975, Leipzig 1976, 7.

4 Ebd.

5 Peter Fischer, Kirchliche Verlage in der DDR, in: Berliner Kirchenreport. Informationsdienst für kirchliche Mitarbeiter v. 20.02.1979.

6 Verordnung über die Entwicklung fortschrittlicher Literatur vom 16.08.1951, in: Gesetzblatt der Deutschen Demokratischen Republik, Berlin v. 27.08.1951, 785.

7 Peter Fischer, a. a. O.

8 Wolfgang Trilling, in: Christine Zauzich, Die Not des Schweigens. Katholiken in der DDR, in: Rheinischer Merkur/Christ und Welt, Nr. 27 v. 06.07.1984.

9 Bekanntmachung über den Bezug des Kirchlichen Amtsblattes, in: Kirchliches Amtsblatt. Ausgabe des Erzbischöflichen Amtes Görlitz vom 01.07.1952, 15

10 Kesseltreiben gegen die kirchliche Jugend. Neue Maßnahmen in der Sowjetzone. Die Säuberungsaktion bei der FDJ, in: Frankfurter Allgemeine Zeitung vom 10.01.1953.

11 Walter Rauch, Presse und Volkstum der Lausitzer Sorben, Würzburg 1959, 163.

12 Anordnung über das Genehmigungsverfahren für die Herstellung von Druck- und Vervielfältigungserzeugnissen vom 20.07.1959, in: Gesetzblatt der Deutschen Demokratischen Republik, T1. 1, Berlin/Ost v. 16.06.1959, 640. Darin heißt es: „Zur Herstellung von Druck- und Vervielfältigungserzeugnissen ist unabhängig von der Zahl der Exemplare sowie von der Art der zur Herstellung benutzten Maschinen, Apparate, Geräte oder Gegenstände eine staatliche Genehmigung (Druckgenehmigung) erforderlich." Von der Genehmigung jedoch ausgenommen sind "Dienstanweisungen, Rundschreiben sowie andere interne Materialien, die im Druck- und Vervielfältigungsverfahren für den inneren Dienstgebrauch der Organe ... hergestellt werden.

13 In eigener Sache, in: begegnung 5/1977, 2.

14 Karl Heinz Ducke, Der Runde Tisch - Was will daran die katholische Kirche?, in: Informationen - Berichte - Materialien aus dem Bereich der Berliner Bischofskonferenz, hg. v. der Pressestelle der Berliner Bischofskonferenz, v. 19.12.1988, 13.

15 Mitarbeit der katholischen Kirche an der Mediengesetzgebung, in: Informationen Berichte - Materialien 4/1990, 13.

16 Beschluß der Volkskammer der Deutschen Demokratischen Republik über die Gewährleistung der Meinungs-, Informations- und Medienfreiheit, in: Media Perspektiven $2 / 1990,126$.

17 Siehe hierzu: ${ }_{n}$ Halle-Magdeburger"-Wochenzeitung gibt es nicht mehr, in: Informationen, hg. v. der Gesellschaft katholischer Publizisten 5/1990, 19. 
18 In eigener Sache, in: begegnung 12/1989, 1.

19 Volk im Neuaufbruch, in: begegnung 12/89, 2.

20 Schreiben des Chefredakteurs der „begegnung“, Horst Walligora, an die Leser der Zeitschrift, Berlin/Ost, Januar 1990.

21 Gottfried Swoboda, Ohne Rücksicht auf Verluste, in: Tag des Herrn v. 01.04.1990, 1.

22 Gottfried Swoboda, Was wird aus dem Tag des Herrn?, in: Tag des Herrn v. 18.11.1990, 1.

23 Ebd., 1f.

24 Brief führender Kirchenvertreter an die Rundfunkverantwortlichen auf dem Territorium der ehemaligen DDR, abgedruckt in: Informationen - Berichte - Materialien Nr. $14 / 1990,17 f$.

25 Ostmitteleuropa: Katholische Medien arbeiten zusammen, in: Kathpress Nr. $120 \mathrm{v}$. 26.06.1990, 9 .

26 Ebd.

27 Ebd. 


\section{SUMMARY}

The journalistic work of the catholic church in the GDR was kept on a tight-rope. Retrospectively it is to be shown just by what means and under what circumstances the catholic church was able to inform their believers. Since October 1989 the catholic work in the media has changed. New opportunities arose, but also difficulties in converting to the market economy. Cooperation with western publishers had to be arranged in order to be able to meet the new requirements. In the field of broadcasting the church is trying very hard to have church editorials installed in the media laws of the five new „Bundesländer". Such editorials, however, also need qualified journalists with religious views. These are rare in the former GDR. Assistance in journalistic education as well as advanced journalistic training is provided by several catholic associations, among others by the „Arbeitsgemeinschaft Katholische Presse (AKP)“ (catholic press association, Anm. d. Ü.).

\section{RÉSUMÉ}

Les travaux de publication de l'Eglise catholique en RDA étaient fortement corsetés. Sous la forme d'une rétrospective, le livre veut montrer par quels moyens et dans quelles conditions l'Eglise catholique pouvait informer ses membres. Depuis octobre 1989 , le travail médiatique des catholiques s'est transformé. De nouvelles chances se sont offertes, mais des difficultés se sont également présentées puisqu'il faut s'adapter à l'économie de marché. On a dû rechercher la coopération d'éditeurs occidentaux afin de pouvoir faire face aux nouveaux défis. Dans le domaine de la radio, l'Eglise s'efforce d'imposer la création des rédactions religieuses dont l'existence serait ancrée dans la législation sur les média des nouveaux 'Bundesländer'. De telles structures ont cependant besoin de journalistes qualifiés aux convictions religieuses solides. Ceux-ci sont plutôt rares dans l'ex-RDA. Différents organismes catholiques, entre autres le cercle de la presse catholique (AKP, Arbeitsgemeinschaft Katholische Presse), ont offert leur aide dans le domaine de la formation des personnels.

\section{RESUMEN}

El trabajo en la opinión pública de la iglesia católica en la RDA tuvo características de un verdadero corsett. En forma de una mirada restrospectiva se señala, con qué medios y bajo qué condiciones la iglesia católica podia informar a sus fieles. Desde octubre de 1989 el trabajo en los medios de la iglesia católica ha cambiado. Se abren nuevas posibilidades, pero también aparecen nuevas dificultades en la adecuación a la economía de mercado. Se tuvo que llegar a acuerdos de cooperación con editoriales occidentales para poder estar a la altura de las nuevas exigencias. En el campo de la radiodifusión, la iglesia se preocupa con gran intensidad de que en las leyes de medios de comunicacón de los cinco nuevos estados federales sean contemplados el derecho a redacciones de las iglesias. Para este tipo de redacciones se necesitan sin embargo periodistas calificados que tengan una convicción religiosa y ésto no es fácil de encontrar en la ex-RDA. Diferentes organizaciones católicas, entre otras la Asociación de Prensa católica (Arbeitsgemeinschaft Katholische Presse) AKP, apoyan la formación y perfeccionamiento en el campo del periodismo. 